\title{
Capturing the Pattern of Physical Activity and Sedentary Behavior: Exposure Variation Analysis of Accelerometer Data
}

\author{
Leon Straker, Amity Campbell, Svend Erik Mathiassen, \\ Rebecca Anne Abbott, Sharon Parry, and Paul Davey
}

\begin{abstract}
Background: Capturing the complex time pattern of physical activity (PA) and sedentary behavior (SB) using accelerometry remains a challenge. Research from occupational health suggests exposure variation analysis (EVA) could provide a meaningful tool. This paper (1) explains the application of EVA to accelerometer data, (2) demonstrates how EVA thresholds and derivatives could be chosen and used to examine adherence to PA and SB guidelines, and (3) explores the validity of EVA outputs. Methods: EVA outputs are compared with accelerometer data from 4 individuals (Study 1a and1b) and 3 occupational groups (Study 2): seated workstation office workers $(n=8)$, standing workstation office workers $(n=8)$, and teachers $(n=8)$. Results: Line graphs and related EVA graphs highlight the use of EVA derivatives for examining compliance with guidelines. EVA derivatives of occupational groups confirm no difference in bouts of activity but clear differences as expected in extended bouts of SB and brief bursts of activity, thus providing evidence of construct validity. Conclusions: EVA offers a unique and comprehensive generic method that is able, for the first time, to capture the time pattern (both frequency and intensity) of PA and SB, which can be tailored for both occupational and public health research.
\end{abstract}

Keywords: accelerometry, physical activity assessment, public health

Physical activity (PA) is typically assessed by selfreport and/or accelerometry. While self-report measures are practical, used widely in surveillance, and continue to provide useful information, ${ }^{1}$ accelerometers are more accurate in capturing time and intensity of PA, with discrepancies between the 2 methods reported to be of meaningful size. ${ }^{2}$ This has led to the increased popularity and reliance on accelerometers in PA research. ${ }^{1,2}$ A range of devices that are small, sophisticated, and relatively inexpensive is currently available; enabling field-based research ${ }^{1,2}$ for long periods of time, even up to a whole year. $^{3}$

Initially, accelerometer use focused on the more accurate recording of the amount of moderate-to-vigorous physical activity (MVPA). ${ }^{4}$ The greater accuracy of accelerometer data has enabled epidemiological research to enhance our understanding of both the links between insufficient MVPA and health risks ${ }^{5}$ and the determinants of MVPA. ${ }^{4}$ Accelerometry has also been

Straker, Campbell, Abbott, Parry, and Davey are with the School of Physiotherapy and Exercise Science, Curtin University, Perth, Western Australia, Australia. Mathiassen is with the Centre for Musculoskeletal Research, Dept of Occupational and Public Health, University of Gävle, Gävle, Gävleborg, Sweden. used to assess efficacy of interventions aimed at increasing MVPA. ${ }^{6}$ The developing understanding of MVPA, its determinants, its effects, and how it can be changed has informed ongoing translation into health promotion and policy development. ${ }^{7}$ The recommendation that adults perform a minimum of 30 minutes of moderate intensity PA on most days and attempt to also perform regular vigorous activities has become well-accepted in most countries, ${ }^{7-9}$ and accelerometry is now also being used to study the compliance with these MVPA recommendations..$^{1,10}$

More recently, not only MVPA but also sedentary behavior (SB) - any waking behavior characterized by an energy expenditure $\leq 1.5$ METs while in a sitting or reclining posture ${ }^{11}$ - has been associated with adverse health outcomes such as obesity, diabetes, metabolic syndrome, and cardiovascular disease. ${ }^{12}$ Sedentariness is also a rising concern in occupational health, in particular in occupations with extensive computer use. ${ }^{13}$ There is increasing evidence that the health effects of sedentariness are independent of time spent performing MVPA $^{14,15}$ and that the adverse health effects of both SB and MVPA need to be studied and addressed separately in both adults ${ }^{14}$ and in children. ${ }^{16}$ As with MVPA research, accelerometer data has also enhanced our understanding of the determinants of SB and enabled the more sensitive 
detection of change following interventions. ${ }^{17}$ However, this enhanced understanding has yet to be translated to guidelines and public health campaigns. ${ }^{17}$ Currently the most widespread guideline related to $\mathrm{SB}$, is the recommendation that parents limit children's screen time (television, DVD, video games, etc) to no more than 2 hours per day. ${ }^{18}$

Until recently, most research articles in this area report total time spent in different levels of activity. ${ }^{6,10,19}$ However, this does not provide information about the pattern of activity exposure across a year, week, day, or any other time window, ${ }^{20}$ despite suggestive evidence that the pattern of activity is important to health. Stronger links between cardiovascular risk indicators and MVPA were found when MVPA exposure was assessed as bouts of at least 10 minutes as opposed to total accumulated MVPA. ${ }^{5,21}$ Similarly prolonged bouts of SB (the authors reported in terms of the number of breaks from SB) have been found to be related to metabolic measures, even after accounting for total accumulated SB, MVPA, and mean intensity of activity. ${ }^{15}$ While the health implications of particular patterns of MVPA and SB are not entirely clear, the evidence suggests robust methods for examining these implications simultaneously should be a priority.

Based on this emerging understanding of the importance of the time pattern of activity, the American College of Sports Medicine updated its recommendation of 30 minutes of MVPA to acknowledge that MVPA should be accumulated in bouts of at least 10 minutes' duration. ${ }^{7}$ SB guidelines are not so well developed; however, it has been suggested on the basis of metabolic well-being that there should be 5 -minute breaks for every 60 minutes of sitting, ${ }^{12}$ and computer use guidelines recommend bouts should not exceed 30-60 minutes before being interrupted with an active break. ${ }^{22,23}$

Accelerometers obviously allow for the determination of the time pattern of SB and MVPA, and this is most commonly represented visually with line graphs of counts per minute over time. ${ }^{2,24}$ While visually effective, this method does not permit data from different subjects, days, or time periods to be documented, compared, or easily translated into guidelines. Reducing the abundant information contained in each day of an individual's accelerometer use into meaningful, analyzable information has been recognized as a significant challenge for continued effectual accelerometer use. ${ }^{2,24}$ Better capture of the time pattern of accelerometer data could lead to an enhanced understanding of the determinants of behavior and of the links between activity and health in both epidemiological and intervention research. Existing examples of methods for examining the time pattern of accelerometer data include assessing time spent in sustained bouts of either MVPA or $\mathrm{SB}^{5,15,21}$ and examination of time spent in MVPA or SB at specific time periods (eg, day or part of day. ${ }^{20}$ More complex examples exist based on nonstandard accelerometry data. ${ }^{25}$ However to our knowledge a method that can assess the time pattern of SB, light activity and MVPA together, based on standard accelerometry data, has not so far been proposed.

While the public health literature has only recently discussed the need for developing generic principles of analyzing patterns of MVPA and SB, this issue has been on the agenda in occupational health research for more than 2 decades. Several methods have been proposed for how to quantify aspects of the time pattern of biomechanical exposures at work, termed "variation" and defined as the change in exposure across time. ${ }^{26}$ These methods include metrics for quantifying the frequency of exposure changes, ${ }^{27}$ the magnitude of exposure changes, ${ }^{18}$ and the similarity of exposure sequences across time. ${ }^{28}$ One method, exposure variation analysis (EVA), ${ }^{29}$ combines the frequency and magnitude aspects of exposure changes across time and has gained considerable attention in the occupational health and ergonomics fields. Thus, EVA has been used extensively to quantify variation in long-term recordings of postures and muscle activity, mainly during occupational work (eg, ${ }^{30-32}$. Basically, EVA reduces a complex time-line of exposure into a 2-dimensional matrix showing combinations of exposure level (in categories) and duration of uninterrupted sequences (in categories). ${ }^{26}$ While most studies using EVA have reported the entire matrix as a description of the characteristics of the investigated exposure, some have processed the matrix into derivative metrics suggested to capture core information on variation. . $^{31,32}$ Thus, EVA offers a generic approach for analyzing "exposure" (in this case, "activity") variation, which could easily be applied to accelerometer recordings of MVPA and SB.

Given the growing recognition of the importance of the pattern of activity for health, the lack of established methods to adequately capture the pattern of accelerometry data, the increasing use of accelerometry in large population studies and intervention studies, and the success of EVA in capturing the pattern of occupational biomechanical exposures, the purpose of this paper is to demonstrate the use and potential of EVA for accelerometer data.

Specifically, this paper aims to (1) explain the application of EVA to accelerometer data for PA and SB (Study 1a), (2) demonstrate how EVA thresholds and derivatives could be chosen and used to examine adherence to MVPA and SB guidelines (Study 1b), and (3) explore the validity of EVA outputs related to PA and SB using known-groups comparison technique (Study 2).

\section{Study 1a: Explanation of Exposure Variation Analysis for Physical Activity and Sedentary Behavior}

\section{Methods}

To illustrate how EVA can be used to interpret accelerometer data, a small study was conducted on 4 healthy university employees. The employees were 
purposively chosen to represent a variety of activity patterns. The participants provided informed consent and the study was approved by the Curtin University Human Research Ethics Committee (PT0154/2010). Participants wore an accelerometer (ActiGraph model GT3X, ActiGraph, LLC, Fort Walton Beach, FL) on a belt over their right anterior superior iliac spine for 1 typical work day and completed a simple activity diary where activity monitor removal and significant activities could be recorded. The accelerometer epoch was set to 1 minute. Only vertical axis data were used, given this is the only axis with established intensity thresholds ${ }^{33,34}$ and the purpose of this paper was to illustrate the utility of EVA. The accelerometer data were downloaded according to the manufacturer's procedures. Data were then exported to prepare line graphs in Microsoft Excel (cpm/time; Microsoft Corporation, Seattle, USA).

The data from 1 participant will be used initially to describe the process of EVA analysis. Figure 1a shows a line graph of accelerometer data for 1 day. Participant 1 was a postdoctoral researcher whose activity pattern was a run before work followed by long periods of uninterrupted sitting at a desk using a computer. The MVPA run is seen as the raised line around 8,000 to $10,000 \mathrm{cpm}$ early in line graph ("A" in Figure 1a), the long periods of $\mathrm{SB}$ can be seen as flat lines along $0 \mathrm{cpm}$ (the first few are labeled "B" in Figure 1a) and the brief bursts of around $500 \mathrm{cpm}$ (some are labeled "C" in Figure 1a).

\section{Results}

EVA was performed using a custom LabVIEW program (LabVIEW 8.6.1, National Instruments, Texas, USA) providing output in minutes. EVA categorized the data for both intensity (device specific count thresholds for sedentary, light, moderate and vigorous; see Study $1 \mathrm{~b}$ methods for further discussion) and the duration of uninterrupted periods at any particular intensity $(0-<5$ minutes, 5-<10minutes, 10 $-<30$ minutes, 30-<60 minutes, and 60+ minutes). Figure $1 \mathrm{~b}$ shows the EVA matrix for the same data shown in Figure 1a. The MVPA run is represented in the vigorous $10-<30$ minutes cell with a total accumulated time of 28 minutes. The long periods of SB are captured in the sedentary $30-<60$ minutes and $60+$ minutes cells with total accumulated time in these cells of 323 and 187 minutes. Figure 1c shows the matrix in graphic form with the MVPA run represented by the small column in the vigorous row $10-<30$ minutes cell ("A") and the long periods of sedentary captured in the large columns in the sedentary row $30-<60$ minutes and $60+$ minutes cells ("B") with the brief bursts in the $0-<5$ row ("C").

Line graphs like Figure 1a are an easy method of presenting an individual's activity, with current recommendations including summarized weekly line graphs. ${ }^{2,24}$ However, it is difficult to compare or interpret the data. In contrast, EVA outlines the participant's activity in meaningful and interpretable groups of activity intensity and duration. Thus, for example, it is easy to determine that while Participant 1 just about meets the MVPA guideline of at least 30 minutes of MVPA she fails to meet the guideline of no uninterrupted bouts of SB greater than 30-60 minutes.

\section{Study 1b: Comparing the Pattern of Physical Activity and Sedentary Behavior of Individuals With Exposure Variation Analysis}

\section{Methods}

Data for Study 1b came from the participant described in Study 1a and 3 additional university employees.

Exposure Variation Analysis Thresholds. To compute accelerometer data EVAs, meaningful activity intensity and duration thresholds are required. Intensity thresholds have typically been based on comparison with energy expenditure, ${ }^{33}$ although comparison with posture (sitting) has been used for SB. ${ }^{18}$ As accelerometer count and energy expenditure are measuring different constructs, the relationship between them varies with the type of activity being performed and is likely to vary between populations due to mechanical and chemical energy efficiency differences. ${ }^{2}$ There is thus considerable inconsistency in the recommendations for count based intensity thresholds. Accelerometer counts for the same input are also known to differ between accelerometer devices ${ }^{33}$ and count thresholds from different devices cannot be directly compared without a translation equation. ${ }^{35}$ Therefore, for the comparison of the 4 illustrative individuals (Study 1a and 1b) measured with ActiGraph accelerometers, we used ActiGraph vertical axis specific thresholds which are widely reported, have reasonable validity, and sit near the center of threshold ranges reported for the ActiGraph accelerometer data $\left(<100 \mathrm{cpm}\right.$ for sedentary, ${ }^{14,24}$ $>100-\leq 1952 \mathrm{cpm}$ for light, ${ }^{34}>1952-5725 \mathrm{cpm}$ for moderate, ${ }^{34,36}$ and $>5725$ cpm for vigorous. ${ }^{34,36}$ For the accelerometer data on the comparison of 3 occupational groups measured with Actical accelerometers (Study 2, to follow), we used equivalent Actical-specific thresholds based on a biological signal derived translation equation (sedentary <91, light 91-1767, moderate 1767-5182, vigorous $>5182) .35$

For the EVA frequency (time period) dimension, we collated uninterrupted sequences in each of the intensity categories into 5 duration levels: $0-<5$ minutes, 5-<10 minutes, 10-<30 minutes, 30-<60 minutes and $60+$ minutes. The rationale for these levels includes that 30 and 60 minutes are periods widely used in SB research, ${ }^{12}$ are considered important for the health impacts of MVPA, ${ }^{37}$ and are used in MVPA guidelines. ${ }^{7}$ The rationale for a 5-minute threshold included the desire to differentiate shorter bouts as these are mentioned 


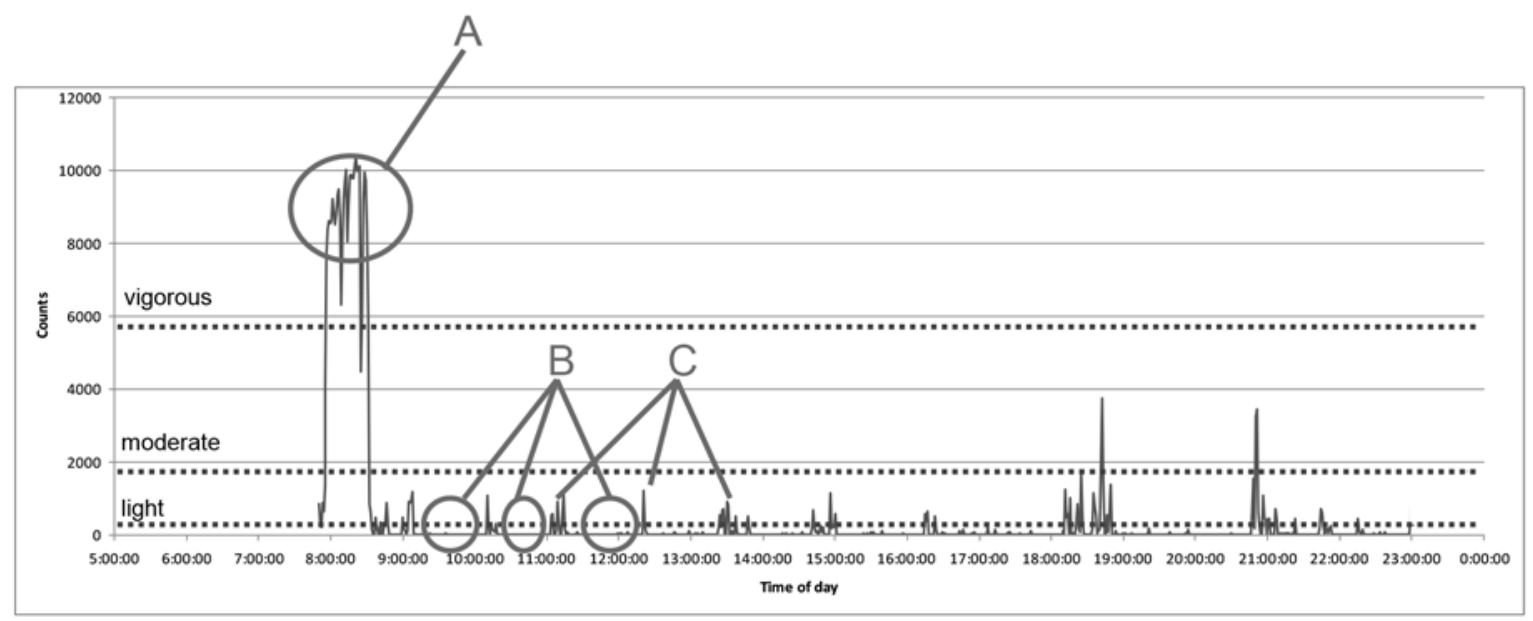

Figure 1a

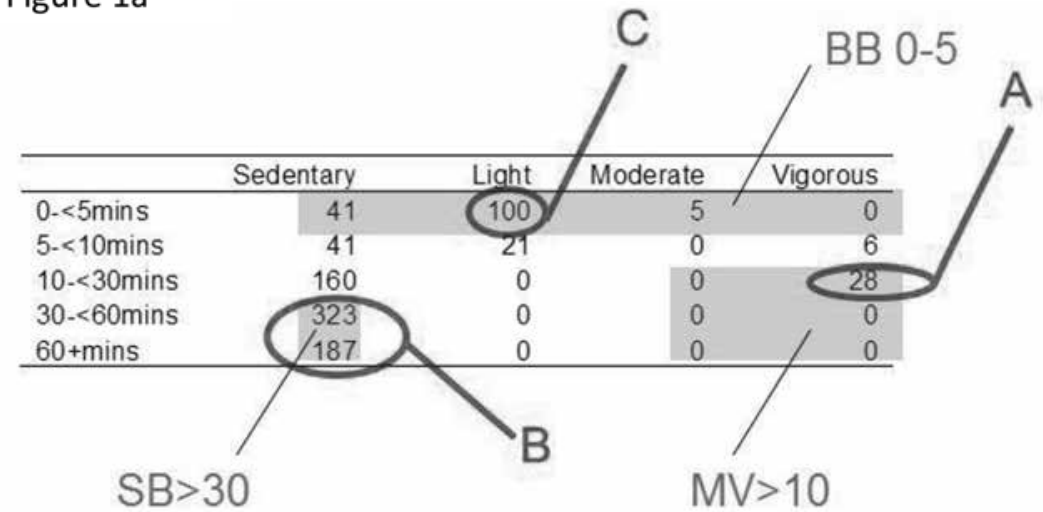

Figure $1 b$

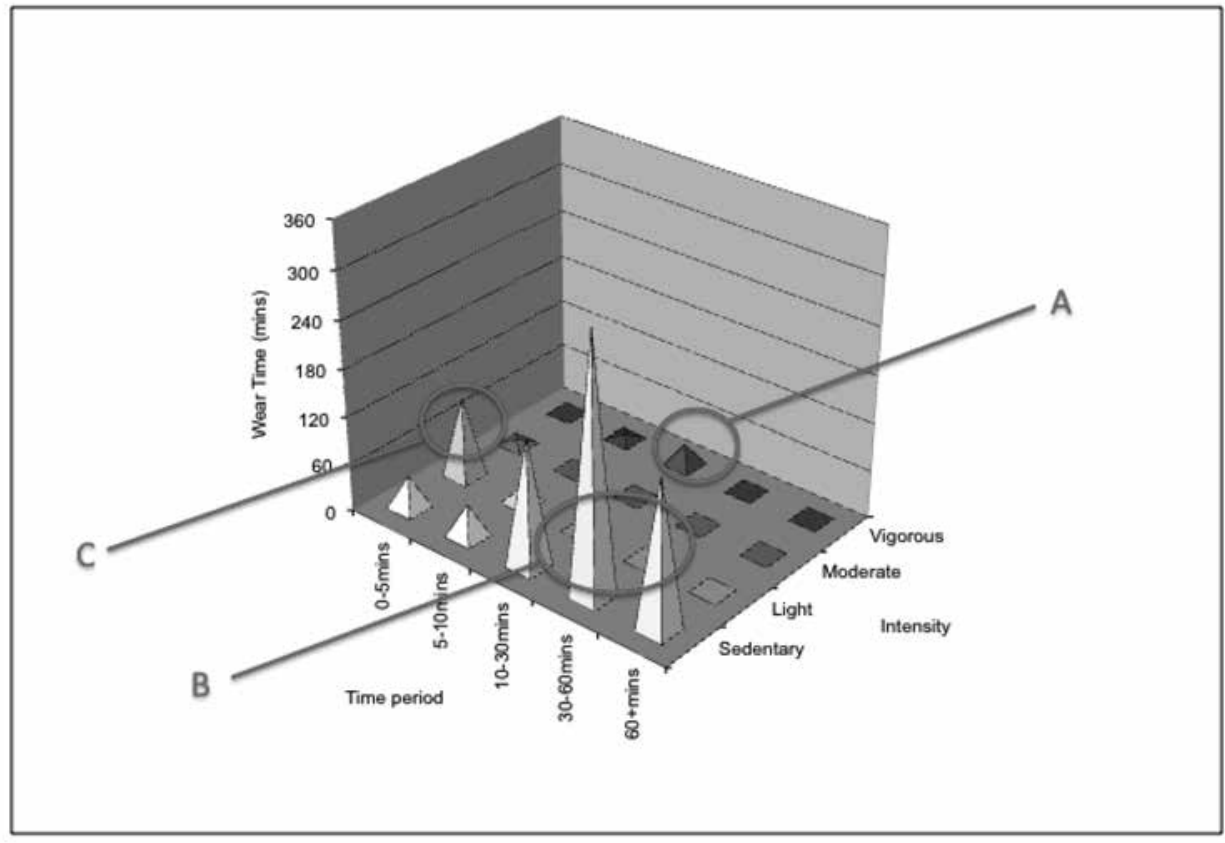

Figure 1c

Figure 1 - A. Line graph of Participant 1 accelerometer data for 1 day showing light, moderate and vigorous intensity thresholds (dotted lines) and highlighting bout of vigorous running (A), bouts of sedentary behavior (SB; B), and brief bursts (C) interrupting SB. B. Exposure variation analysis (EVA) matrix in minutes for Participant 1 accelerometer data for 1 day highlighting capture of bout of vigorous running (A), long bouts of sedentary behavior (B) and brief bursts (C) interrupting SB (shaded areas show cells summed for EVA derivatives MV >10, SB >30, BB 0-5). C. Graph of EVA matrix in minutes for Participant 1 accelerometer data for 1 day highlighting capture of bouts of moderate to vigorous activity, including the 10-30 minutes of vigorous running (A), bouts longer than 30 minutes of SB (B), and brief burst creating activity variation, among which many involve light PA (C). 
in recommendations, ${ }^{12,23}$ and they may be a proxy for breaking up sustained periods in the exposure time line.

EVAs presented for participants 1-4 have cell magnitudes (column heights) reported in minutes of wear time to enable simple comparison with guidelines. However, cell magnitudes can be reported as a percentage of wear time to account for differences between participant day length (eg, a work day vs a whole 14-18-hours-awake day that includes leisure) in group analyses. Outputs in time proportion terms have been the dominant approach in studies using EVA to date.

Exposure Variation Analysis Derivatives. We propose that while EVA provides a generic approach to characterizing the pattern of accelerometer determined activity, derivatives of the EVA matrix can be developed for particular purposes. To illustrate this, 3 derivatives have been calculated from EVA. The first 2 are based on widely used guidelines, and the third is based on the occupational health principle of encouraging variation/ nonsustained activity.

"Bout MVPA" (MV >10) shows the time or proportion of time performing MVPA sustained for durations greater than 10 minutes and is calculated by summing 6 cells (moderate $10-<30,30-<60$, and $60+$; and vigorous $10-<30,30-<60$, and $60+)$. As previously mentioned, MVPA accumulated in bouts of greater than 10 minutes is more strongly related to health outcomes than overall MVPA exposure. ${ }^{21}$ Participant 1 accumulated 28 minutes of bout MVPA (see MV $>10$ in Figure 1b).

"Sustained SB" (SB >30) shows the time or proportion of time in SBs sustained for durations greater than 30 minutes and is calculated by summing 2 cells (sedentary 30-<60 and 60+). Sustained SB is known to be associated with increased health risks. ${ }^{14}$ Participant 1 accumulated 510 minutes of sustained SB (see SB > 30 in Figure 1b).

"Brief bursts" (BB 0-5) shows time or proportion of time in any intensity level of activity which only lasts for less than 5 minutes and is calculated by summing 4 cells (sedentary, light, moderate and vigorous $0-<5$ ). Variation in biomechanical and physiological exposures at work is believed to be associated with reduced risk of musculoskeletal disorders. ${ }^{26}$ Participant 1 accumulated 146 minutes of this potentially healthy behavior (see BB $0-5$ in Figure $1 b$ ).

\section{Results}

Figures 2a, 2b, and 2c show line graphs of accelerometer data for 1 day for the 3 other university employees. Figures 3a, 3b, and 3c present EVA graphs for the same data. Participant 2 was a teaching laboratory assistant responsible for setting out equipment for classes and general facility maintenance. The activity pattern for Participant 2 was for continual variation between sedentary and light activity, seen in the lack of flat lines in the line graph (Figure 2a) and the lack of EVA graph (Figure 3a) columns in the sedentary $30-<60$ and $60+$ cells, as well as in the clustering of columns in short durations of sedentary and light activity.

Participant 3 was a resource officer responsible for an equipment library and helping with shifting equipment. Their activity pattern was for sedentary periods interspersed with brief light activity. In the line graph, there are flat line periods around $0 \mathrm{cpm}$, but these are interspersed with spikes of activity often around 1500 cpm in magnitude (Figure 2b), reflected in the tall column in the sedentary $10-<30$ minute cell and a medium height column in the light $0-<5$ minute cell of the EVA graph (Figure 3b).

Participant 4 was another post doctorate researcher whose activity pattern was for many breaks in sedentary activity during the day as well as sustained periods of MVPA both before (a run) and after ( 2 walks) work. In the line graph (Figure 2c) these show as intermittent spikes in the flat lines around $0 \mathrm{cpm}$ periods with sustained periods with cpm over 4000 at the start and end of the day. In the EVA graph (Figure 3c) these show as no column in the sedentary $60+$ cell and small columns in the moderate and vigorous rows.

Table 1 shows the mean and standard deviation cpm for all 4 participants in the initial study. The means show Participants 1 and 3 are less active and Participant 4 is most active, but obviously this provides no information on the pattern of PA or SB. The standard deviations of accelerometer counts across a day suggest Participants 1 and 4 have a similar variation in activity, but this seems misleading given the patterns of activity shown in the line graphs. Table 1 also shows the minutes each participant accumulated within each intensity level. While this clearly shows the high SB exposure of Participant 1 and high MVPA exposure of Participant 4, it does not provide information about whether the SB and MVPA was accumulated in bouts known to be important to health. ${ }^{13,21}$

From the EVA derivatives presented in Table 1 it can be seen that Participant 4 clearly exceeds the guideline for accumulating more than 30 minutes MVPA in bouts of at least 10 minutes, Participant 1 comes close to meeting this guideline and Participants 2 and 3 do not meet the guideline. Participant 2 clearly meets the guideline for avoiding periods of 30minutes or more of SB, while Participant 1 has a potentially harmful exposure of 510 minutes in such periods. Participant 2 also has the greatest exposure (360 minutes) to brief bursts which may be beneficial to health.

The illustration above shows how EVA can capture the pattern of PA and SB of 4 individuals and allow interpretation of activity in terms of meeting guidelines and exposure to potentially harmful and potentially health promoting activity patterns. Together, these provide evidence for the potential utility of EVA. The following section (Study 2) explicitly examines the utility of EVA in capturing group data and additionally examines aspects of validity. 


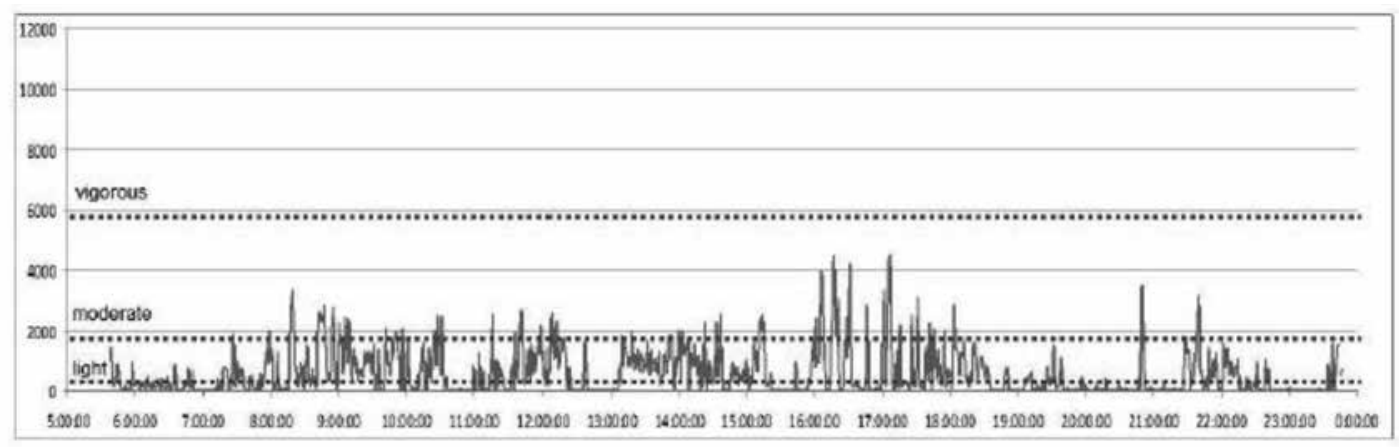

$2 a$

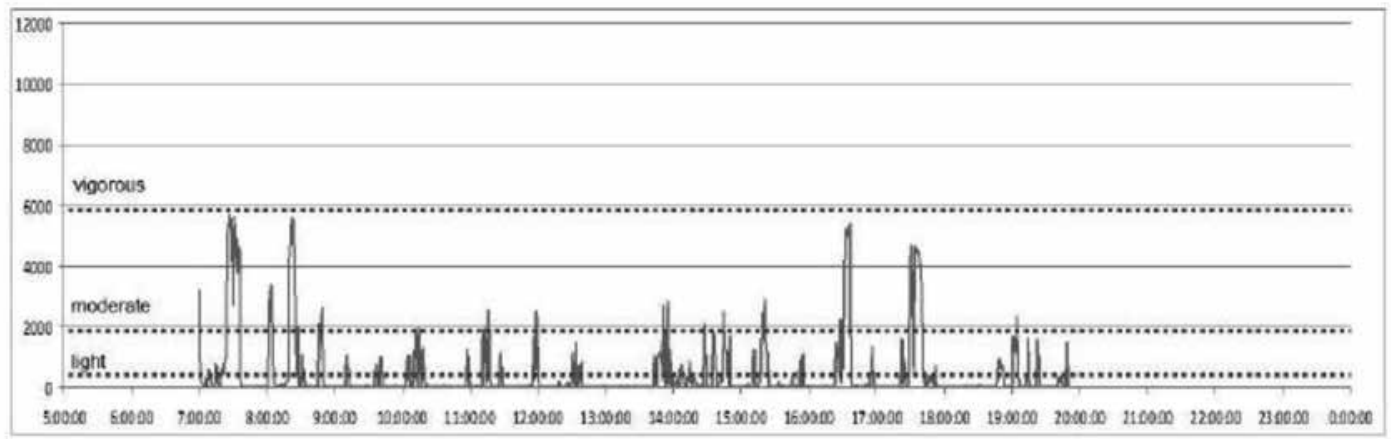

$2 \mathrm{~b}$

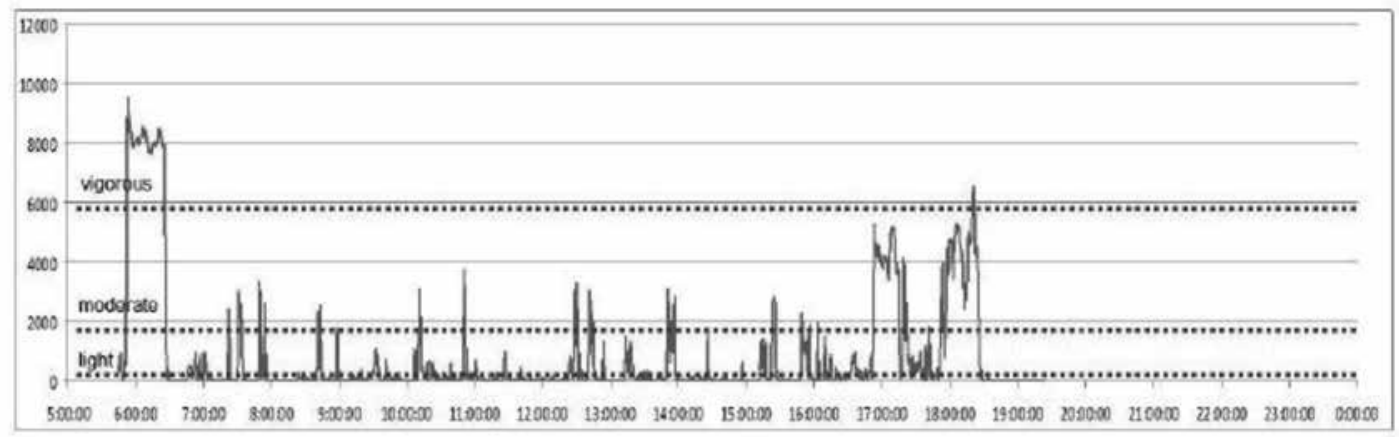

2c

Figure 2 - Accelerometer output (counts/minute) for a whole day from Participant 2 (2a), Participant 3 (2b), and Participant 4 (2c).

\section{Study 2: Comparing the Pattern of Physical Activity and Sedentary Behavior of Groups With Exposure Variation Analysis}

\section{Methods}

To explore the utility and validity of the EVA derivatives proposed above, data from a study of 3 groups of workers were used. A common approach to establishing a higher level of evidence than face validity is to compare groups with assumed differences to determine construct validity - the known-groups comparison technique. Knowngroups comparison anticipates that specific groups are different based on a specific outcome, thus the measure should be sensitive to these differences. ${ }^{38}$ Therefore, 3 groups - assumed to be different in their activity levels through the nature of their work-were chosen: 8 (3 female) office workers who used traditional seated computer workstations, 8 (3 female) office workers using standing computer workstations, and 8 (6 female) primary 


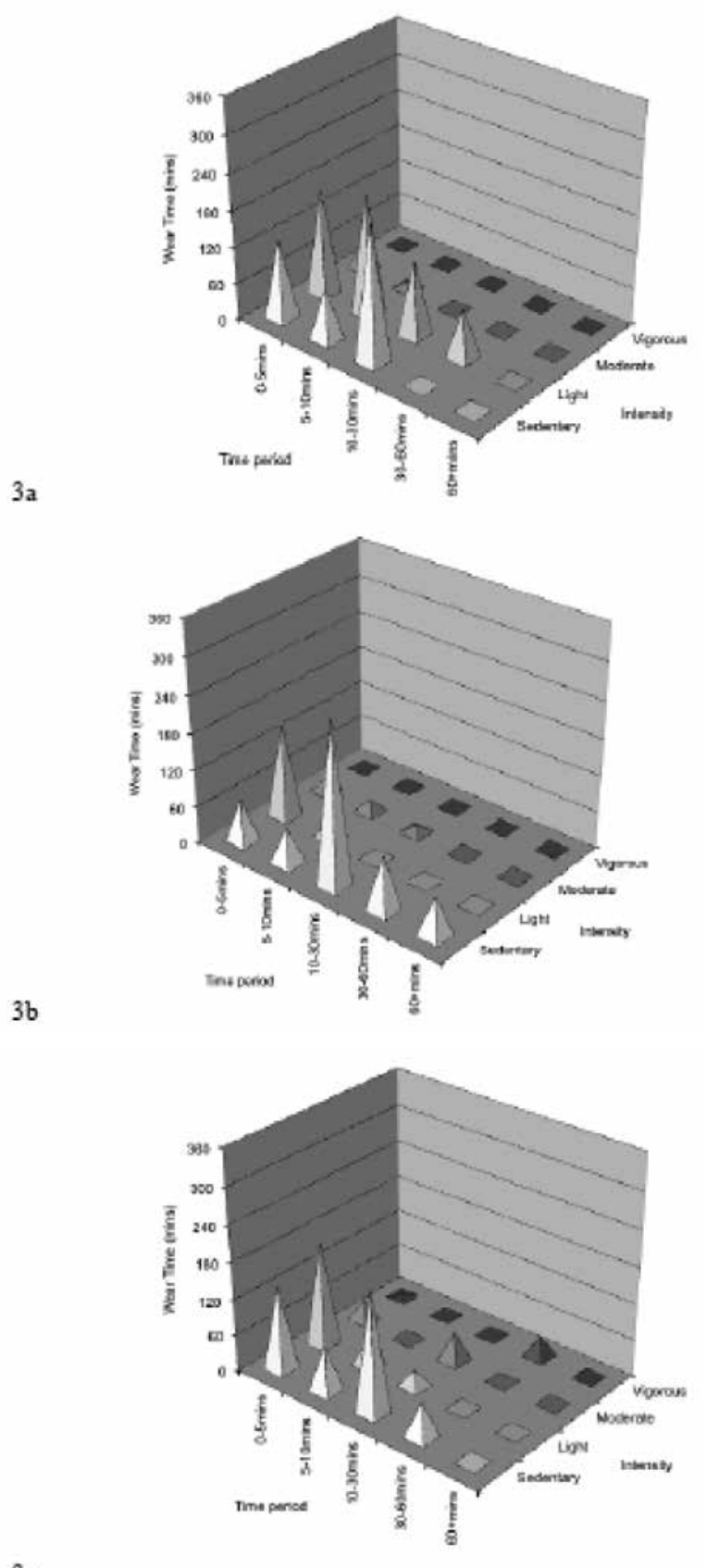

$3 \mathrm{c}$

Figure 3 - Exposure variation analysis graphs based on accelerometer output (counts/minute) from a whole day for Participant 2 (3a), Participant 3 (3b), and Participant 4 (3c), in minutes.

and high school teachers. Differences in time spent sitting and standing have been found in call center workers with sitting or sit-stand workstation designs using inclinometers, ${ }^{39}$ and in pilot work where teachers were observed to break up periods of sitting more frequently than office workers. The participants provided informed consent and the study was approved by Curtin University Human Research Ethics Committee (HR20, 2007).
Participants wore an Actical (Respironics, Bend, OR) accelerometer on a belt over the right anterior superior iliac spine for 4 work days and completed a simple activity diary where activity monitor removal and significant activities could be recorded. The Actical provides a single omnidirectional output most closely related to ActiGraph vertical axis output. ${ }^{35}$ The accelerometer epoch was set to 1 minute.

Accelerometer data were downloaded according to the manufacturer's procedures and visually inspected for quality control. EVA analysis was performed using a custom LabVIEW 8.6.1 program using the intensity and duration categories outlined in Study $1 \mathrm{~b}$ methods above. The LabVIEW output provided an EVA matrix for each day for each worker. Using Microsoft Excel, an average EVA matrix for each worker was calculated (each cell averaged).

\section{Results}

The age and BMI of the 3 groups were similar: seated office workers, standing office workers and teachers had a mean (SD) age of 36.0 (7.4) years, 37.9 (9.1) years, and 40.6 (7.7) years and mean (SD) BMI of 25.2 (5.0), 25.3 (3.3), and 23.3 (1.7), respectively. An average EVA for each occupational group was calculated for illustrative purposes using the same averaging method on the mean cell values for subjects in each group. From group average EVA graphs (Figures $4 \mathrm{a}-\mathrm{c}$ ) it is clear that the main differences between groups appeared to be in the sedentary and light categories, and not in the moderate or vigorous categories. The seated office work groups were sedentary for most of their work day, with large proportions of the day in periods exceeding 30 and 60 minutes. For the standing office worker group the obvious difference is the much lower exposure to sedentary periods exceeding 60 minutes. For the teacher group the difference is less exposure to sedentary periods exceeding both 30 and 60 minutes as well as a greater proportion of the day in brief $(0<5$ minutes $)$ bursts of light activity.

The data were examined for group differences in mean values and exposure variability of each of the 3 proposed EVA derivatives. For each occupational group, the variance between subjects, $s_{s}^{2}$, and variance between days within subject, $s_{d}^{2}$, was estimated using ANOVA in a standard 1-way hierarchical model with random factors. ${ }^{40}$ Contrasts, $C_{G}$, between pairs of groups were assessed using the index $C_{G}=\mathrm{MS} \Delta_{\mathrm{G}} /\left(\mathrm{MS} \Delta_{\mathrm{G}}+s_{s}^{2}\right)$, where $\mathrm{MS} \Delta_{\mathrm{G}}$ is the mean squared deviation of the 2 exposure mean values from their common mean, ${ }^{41}$ adjusted for the effect of within-group exposure variability. $C_{G}$ measures the diversity of groups (in casu, 2) on a scale from 0 (groups cannot be discriminated) to 1 (groups differ in exposure while subjects within the groups have identical exposures). The size of $C_{G}$ is closely related to the number of subjects needed to obtain a statistically significant difference between groups. ${ }^{42}$ Thus, for the present case of 8 subjects in each group, standard $P$ values for the difference between each pair of groups were also assessed 
using least square differences post hoc tests in a univariate general linear model in SPSS (v19).

As shown in Table 2, the mean exposure to bout MVPA (MV >10) was small for all groups, and the groups did not differ to any particular extent $\left(F_{2,21}=1.79, P=\right.$ .191). This lack of diversity was expected since none of the 3 occupations required MVPA in the job. Only the standing office workers exhibited a clear exposure variability between subjects and days, mainly due to 1 subject having both a considerably larger mean than the other 7 , and a large variability between days in MVPA. In contrast, for the sustained SB (SB > 30) the groups clearly differed $\left(F_{2,21}=9.32, P=.001\right)$, with an expected largest mean value for the seated office workers and lowest mean for

Table 1 Accelerometer Statistics for 1 Day From 4 Participants

\begin{tabular}{|c|c|c|c|c|}
\hline & Participant 1 & Participant 2 & Participant 3 & Participant 4 \\
\hline \multicolumn{5}{|c|}{ Accelerometer count (cpm) } \\
\hline Mean & 427 & 570 & 435 & 864 \\
\hline Standard deviation & 1726 & 752 & 1052 & 1918 \\
\hline \multicolumn{5}{|c|}{ Intensity levels (minute[\%]) } \\
\hline Sedentary & $752[82.5]$ & $440[40.5]$ & $543[70.5]$ & $468[57.1]$ \\
\hline Light & $121[13.3]$ & $579[53.3]$ & $175[22.7]$ & $236[28.8]$ \\
\hline Moderate & $5[0.5]$ & $67[6.2]$ & $52[6.8]$ & $80[9.8]$ \\
\hline Vigorous & $34[3.7]$ & $0[0]$ & $0[0]$ & $36[4.4]$ \\
\hline \multicolumn{5}{|c|}{ EVA derivatives (minute [\%]) } \\
\hline $\mathrm{MV}>10$ & $28[3.1]$ & $0[0]$ & $12[1.6]$ & $81[9.9]$ \\
\hline $\mathrm{SB}>30$ & $510[55.9]$ & $0[0]$ & $151[19.6]$ & 57 [7] \\
\hline BB $0-5$ & $146[16]$ & $360[33.1]$ & $240[31.2]$ & $344[42]$ \\
\hline
\end{tabular}

Abbreviations: EVA, exposure variation analysis; MV, moderate-vigorous; SB, sedentary behavior; BB, brief bursts.

Table 2 Mean Values, Variance Components, and Contrasts for the 3 Exposure Variation Analysis Derivatives MV > 10, SB > 30, and BB 0-5 in Each of the 3 Occupational Groups

\begin{tabular}{|c|c|c|c|c|c|c|}
\hline & $\begin{array}{l}\text { A. Seated office } \\
\text { workers }\end{array}$ & $\begin{array}{l}\text { B. Standing office } \\
\text { workers }\end{array}$ & C. Teachers & $A$ vs $B$ & A vs $C$ & $B$ vs $C$ \\
\hline \multicolumn{7}{|l|}{$\mathrm{MV}>10$} \\
\hline Mean & 0.9 & 2.6 & 0.5 & & & \\
\hline $\mathrm{s}_{\mathrm{s}}^{2}$ & 1.0 & 13.0 & $0^{*}$ & & & \\
\hline $\mathrm{s}_{\mathrm{d}}^{2}$ & 1.5 & 8.7 & 1.0 & & & \\
\hline $\mathrm{C}_{\mathrm{G}}$ & - & - & - & 0.02 & $0 *$ & 0.08 \\
\hline$P$ & - & - & - & .179 & .681 & .085 \\
\hline \multicolumn{7}{|l|}{$\mathrm{SB}>30$} \\
\hline Mean & 37.3 & 25.7 & 15.7 & & & \\
\hline $\mathrm{s}_{\mathrm{s}}^{2}$ & 129.9 & 53.6 & 42.2 & & & \\
\hline $\mathrm{s}_{\mathrm{d}}^{2}$ & 66.6 & 76.6 & 39.1 & & & \\
\hline $\mathrm{C}_{\mathrm{G}}$ & - & - & - & 0.23 & 0.56 & 0.30 \\
\hline$P$ & - & - & - & .023 & $<.001$ & .077 \\
\hline \multicolumn{7}{|l|}{ BB $0-5$} \\
\hline Mean & 21.8 & 26.8 & 34.6 & & & \\
\hline $\mathrm{s}_{\mathrm{s}}^{2}$ & 12.2 & 6.9 & $0^{*}$ & & & \\
\hline $\mathrm{s}_{\mathrm{d}}^{2}$ & 20.6 & 16.3 & 26.3 & & & \\
\hline $\mathrm{C}_{\mathrm{G}}$ & - & - & - & 0.36 & 0.88 & 0.83 \\
\hline$P$ & - & - & - & .007 & $<.001$ & $<.001$ \\
\hline
\end{tabular}

\footnotetext{
* Negative value replaced by 0 .
}

Abbreviations: $s_{s}^{2}, s_{d}^{2}$, variance between subjects within group and between days within subject, respectively; $C_{G}$, contrast between groups; MV, moderate-vigorous; $\mathrm{SB}$, sedentary behavior; $\mathrm{BB}$, brief bursts. 
the teachers. Exposure variability was obvious in all 3 groups both between subjects and between days (noting that individual days without exposure to long seated periods, $\mathrm{SB}>30$, enable subjects to have an average exposure over 4 days of less than 30 minutes.) The largest differences between the 3 groups were found for brief bursts (BB $0-5 ; F_{2,21}=28.24, P<.001$ ). As expected, teachers exhibited the largest occurrence of these shortterm variations in activity, and seated office workers the least. Groups were very homogeneous for this variable, as shown by small between-subject variances, and subjects behaved consistently across days, as shown by a quite small between-days variance.

Thus, the comparison of the 3 groups using our EVA derivatives confirmed expectations on differences and similarities, and demonstrated the construct validity of those derivatives. In addition, $\mathrm{SB}>30$ and $\mathrm{BB} 0-5$ were sensitive to differences between quite small groups suggesting adequate sensitivity for use in intervention studies. Whether MV $>10$ is also sufficiently sensitive remains to be documented in studies assessing groups known to differ in the occurrence of MVPA.

\section{Discussion}

Accelerometry has proven to be a valuable tool for PA and SB assessment and has enabled a developing understanding of the health impacts of SB and MVPA, including the importance of the time pattern of activity. However, to date analyses accounting for this pattern have largely been restricted to estimating bouts of inactivity or activity. While all the EVA cell values and derivatives could be calculated separately, we propose that EVA is particularly useful as it can be used to assimilate data, at both the individual or group level, and to provide a comprehensive assessment of MVPA, light activity, and SB pattern, assessing both frequency and level of intensity together. Reducing the abundant information from accelerometry into meaningful, analyzable information has long been recognized as a significant challenge for continued effectual accelerometer use,, 24 and we believe that not only does EVA achieve this, but is the first approach to do so simultaneously for MVPA, light activity, and SB with flexibility. This paper has explained the use of EVA for accelerometer data using examples from 4 individual workers and 3 occupational groups, demonstrating its utility and construct validity using the known-groups comparison technique.

The thresholds and derivatives outlined are not definitive, but provide examples of how EVA can be used for PA research. The MV >10 derivative is directly equivalent to prior analyses and thus the validity of this derivative in terms of being related to health indicators is the same as prior measures of MVPA bouts greater than 10 minutes. ${ }^{5,7,21}$ In contrast, $\mathrm{SB}>30$ and $\mathrm{BB} 0-5$ are novel examples of derivatives that could be aligned to $\mathrm{SB}$ guidelines. Prior research on sustained SB has characterized breaks from $\mathrm{SB}^{14,19}$ but has not specifically addressed duration of SB bouts.

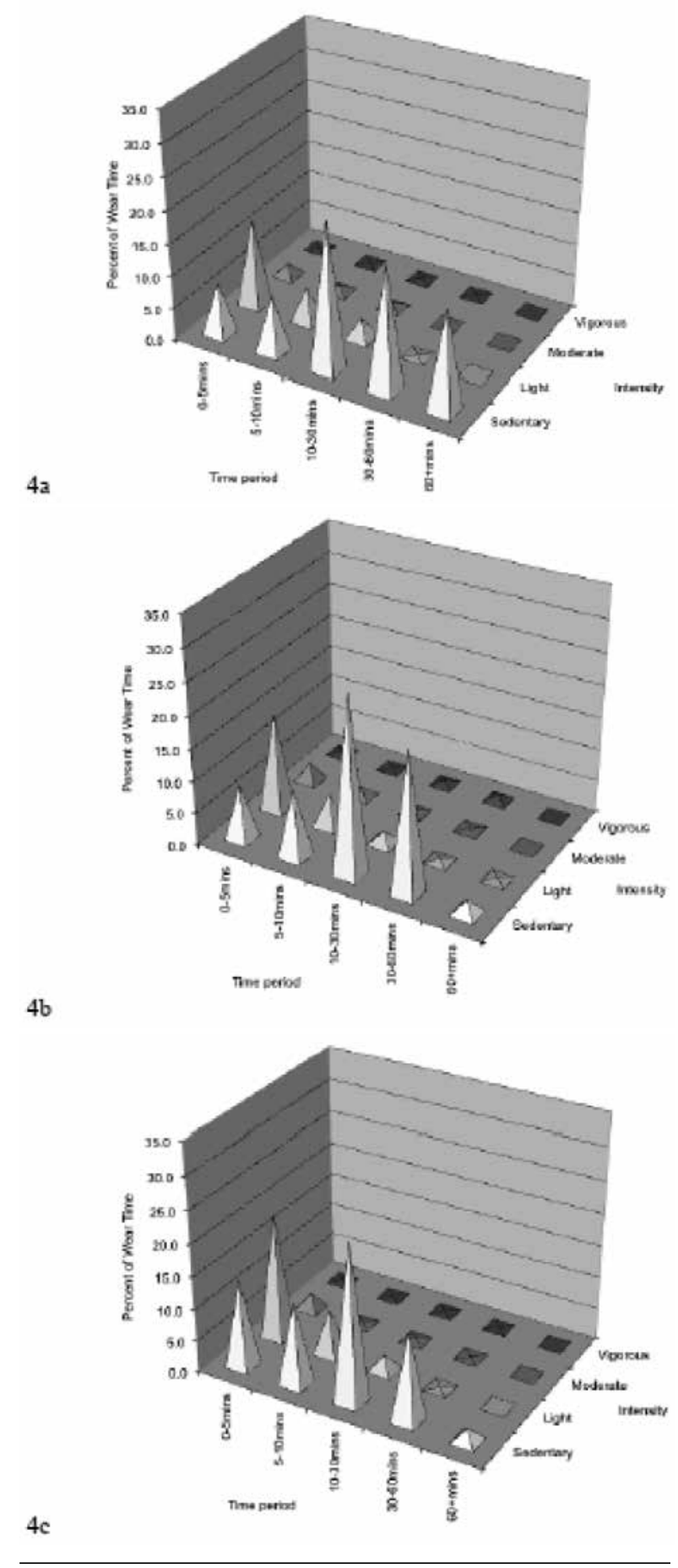

Figure 4 - Exposure variation analysis graphs based on categorized accelerometer output (counts/minute) from (a) seated office workers, (b) standing office workers, and (c) teachers in proportion of wear time.

The major advantage of EVA is its ability in a single analysis to simultaneously capture the time pattern of activity at various levels of intensity according to the choice of the researcher. The EVA presented here used 4 levels of intensity—sedentary, light, moderate, and 
vigorous - but EVA could be designed with any number of categories including dichotomous data such as sitting vs standing. Analyses of temporal patterns of dichotomous exposures has been presented in several studies of occupational muscle activity ("gap" analysis 43 ; "burst" analysis ${ }^{44}$ ), showing that a temporal analysis even at this simplified level can give important information on health outcomes. A further advantage of EVA is that the matrix and derivatives can be adjusted to match future refinement of PA guidelines. The derivatives are also readily translated into public health messages and compliance assessed in relevant groups, for example, as when stating the proportion of people meeting the suggested guideline "avoid sustained periods of SB greater than 30 minutes."

In this paper, we used data at a 1-minute resolution from a single vertical or omnidirectional plane. EVA could easily be used with triaxial and shorter epoch data, which may provide further valuable information. By using different matrix classifications of time sequences adapted to the accelerometer sampling frequency, EVA can be used in studies devoted to understanding the occurrence and effects of even very short epochs of changed behavior. Thus in future studies on the relationships between movement and health, EVA matrix formats can be designed specifically to suit the variables of interest. Consideration of nonwear time definitions should also be given when determining EVA duration levels to avoid misclassification.

EVA can be used to examine likely determinants of activity such as time of day, place, and task by stratifying data accordingly and computing an EVA and its derivatives for each stratum of data. While this does not fully capture the real-time aspects of activity, it could, for example, compare activity patterns before and after school, during work and during leisure, at home and in the community, and during computer-based tasks and non-information-technology tasks.

It is likely that the important aspects of the pattern of PA and SB, and thus appropriate EVA derivatives, are different for different health outcomes. Thus, the optimal pattern of activity to reduce diabetes risk may be low SB $>30$ exposure, whereas the optimal pattern of activity to reduce myocardial infarct risk may be high $\mathrm{MV}>10$ and BB 0-5 exposure. By enabling the simultaneous analysis of duration and magnitude of activity, EVA derivatives could be developed into metrics reflecting compliance with activity prescriptions targeted to the health risks of individuals.

In conclusion, while EVA has already demonstrated utility in occupational electromyographic and posture research, we claim that it also offers a comprehensive generic method for capturing the time pattern of activity, which can be tailored for a wider use in occupational and public health research.

\section{Acknowledgments}

The authors would like to thank the participants and their organizations for enabling data collection. LS was funded by an Australian National Health and Medical Research Council senior research fellowship, AC by a Curtin University research fellowship, and RA by a Centre for Musculoskeletal Research, University of Gävle research fellowship.

\section{References}

1. Hallal PC, Andersen LB, Bull FC, Guthold R, Haskell W, Ekelund U; Lancet Physical Activity Series Working Group. Global physical activity levels: surveillance progress, pitfalls, and prospects. Lancet. 2012;380(9838):247257. PubMed doi:10.1016/S0140-6736(12)60646-1

2. Esliger DW, Tremblay MS. Physical activity and inactivity profiling: the next generation. Can J Public Health. 2007;98(Suppl 2):S195-S207. PubMed

3. Aoyagi Y, Park H, Park S, Shephard RJ. Habitual physical activity and health-related quality of life in older adults: interactions between the amount and intensity of activity (the Nakanojo Study). Qual Life Res. 2010;19(3):333-338. PubMed doi:10.1007/s11136-010-9588-6

4. Trost SG, Saunders R, Ward DS. Determinants of physical activity in middle school children. Am J Health Behav. 2002;26:95-102. PubMed doi:10.5993/AJHB.26.2.2

5. Atienza AA, Moser RP, Perna F, et al. Self-reported and objectively measured activity related to biomarkers using NHANES. Med Sci Sports Exerc. 2011;43:815-821. PubMed doi:10.1249/MSS.0b013e3181fdfc32

6. Ainsworth BE, Bassett DR, Strath SJ, et al. Comparison of three methods for measuring the time spent in physical activity. Med Sci Sports Exerc. 2000;32:S457-S464. PubMed doi:10.1097/00005768-200009001-00004

7. Haskell WL, Lee IM, Pate RR, et al. Physical activity and public health: updated recommendation for adults from the American College of Sports Medicine and the American Heart Association. Med Sci Sports Exerc. 2007;39:14231434. PubMed doi:10.1249/mss.0b013e3180616b27

8. Bull FC and the Expert Working Groups. Physical Activity Guidelines in the UK: review and recommendations. School of Sport, Exercise and Science, Loughborough University, May 2010, px-xii

9. Department of Health and Aged Care. National Physical Activity Guidelines. Canberra, Australia: The Australian Federal Government, 1999; http://www.health.gov.au/ internet/main/publishing.nsf/content/phd-physical-activity-adults-pdf-cnt.htm. Accessed December 2, 2011.

10. Schoenborn CA, Stommel M. Adherence to the 2008 adult physical activity guidelines and mortality risk. Am J Prev Med. 2011;40:514-521. PubMed doi:10.1016/j. amepre.2010.12.029

11. Sedentary Behavior Research Network. Letter to the editor: standardized use of the terms "sedentary" and "sedentary behaviours." Appl Physiol Nutr Metab 2012, 37:540-542.

12. Owen N, Bauman A, Brown W. Too much sitting: a novel and important predictor of chronic disease risk? Br J Sports Med. 2009;43:81-83. PubMed doi:10.1136/ bjsm.2008.055269

13. Straker L, Mathiassen SM. Increased physical work loads in modern work: a necessity for better health and performance? Ergonomics. 2009;52:1215-1225. PubMed doi:10.1080/00140130903039101 
14. Healy GN, Dunstan DW, Salmon J, Cerin E, Shaw JE, Zimmet PZ. Breaks in sedentary time: beneficial associations with metabolic risk. Diabetes Care. 2008;31:661666. PubMed doi:10.2337/dc07-2046

15. Healy GN, Matthews CE, Dunstan DW, Winkler EAH, Owen N. Sedentary time and cardio-metabolic markers in US adults: NHANES 2003-06. Eur Heart J. 2011;32:590597. PubMed doi:10.1093/eurheartj/ehq451

16. Tremblay MS. Major initiatives related to childhood obesity and physical inactivity in Canada: the year in review. Can J Public Health. 2007;98:457-459. PubMed

17. Tremblay MS, Colley RC, Saunders TJ, Healy GN, Owen N. Physiological and health implications of a sedentary lifestyle. Appl Physiol Nutr Metab. 2010;35:725-740. PubMed doi:10.1139/H10-079

18. Ciccarelli M, Straker L, Mathiassen SE, Pollock C. ITKids part II: variation of postures and muscle activity in children using different information and communication technologies. Work. 2011;38:413-427. PubMed

19. Matthews CE, Chen KY, Freedson PS, et al. Amount of time spent in sedentary behaviors in the United States, 2003-2004. Am J Epidemiol. 2008;167:875-881. PubMed doi:10.1093/aje/kwm390

20. Metzger JS, Catellier DJ, Evenson KR, Treuth MS, Rosamond WD, Siega-Riz AM. Patterns of objectively measured physical activity in the United States. Med Sci Sports Exerc. 2008;40:630-638. PubMed doi:10.1249/ MSS.0b013e3181620ebc

21. Murphy MH, Blair SN, Murtagh EM. Accumulated versus continuous exercise for health benefit: a review of empirical studies. Sports Med. 2009;39:29-43. PubMed doi:10.2165/00007256-200939010-00003

22. Straker L, Maslen B, Burgess-Limerick R, Johnson P, Dennerlein J. Evidence-based guidelines for the wise use of computers by children: physical development guidelines. Ergonomics. 2010;53:458-477. PubMed doi:10.1080/00140130903556344

23. Swedish Work Environment Authority. Work with display screen equipment. Provisions of the Swedish National Board of Occupational Safety and Health AFS. Stockholm: Swedish Work Environment Authority, 1998. Available from: Swedish Work Environment Authority, p. 119-121.

24. Esliger DW, Copeland JL, Barnes JD, Tremblay MS. Standardizing and optimizing the use of accelerometer data for free-living physical activity monitoring. $J$ Phys Act Health. 2005;3:366-383.

25. Paraschiv-Ionescu A, Perruchoud C, Buchser E, Aminian $\mathrm{K}$. Barcoding human physical activity to assess chronic pain conditions. PLOS ONE. 2012;7(2):e32239. PubMed doi:10.1371/journal.pone.0032239

26. Mathiassen SE. Diversity and variation in biomechanical exposure: what is it, and why would we like to know? Appl Ergon. 2006;37:419-427. PubMed doi:10.1016/j. apergo.2006.04.006

27. Kilbom A, Persson J. Work technique and its consequences for musculoskeletal disorders. Ergonomics. 1987;30:273279. PubMed doi:10.1080/00140138708969706

28. Mathiassen SE, Moller T, Forsman M. Variability in mechanical exposure within and between individuals performing a highly constrained industrial work task. Ergonomics. 2003;46:800-824. PubMed doi:10.1080/0014013031000090125

29. Mathiassen SE, Winkel J. Quantifying variation in physical load using exposure-vs-time data. Ergonomics. 1991;34:1455-1468. PubMed doi:10.1080/00140139108964889

30. Bao S, Mathiassen SE, Winkel J. Ergonomic effects of a management-based rationalization in assembly work - a case study. Appl Ergon. 1996;27:89-99. PubMed doi:10.1016/0003-6870(95)00063-1

31. Straker LM, Coleman J, Skoss R, Maslen BA, Burgess-Limerick R, Pollock CM. A comparison of posture and muscle activity during tablet computer, desktop computer and paper use by young children. Ergonomics. 2008;51:540555. PubMed doi:10.1080/00140130701711000

32. Anton D, Cook TM, Rosecrance JC, Merlino LA. Method for quantitatively assessing physical risk factors during variable noncyclic work. Scand J Work Environ Health. 2003;29:354-362. PubMed doi:10.5271/sjweh.742

33. Puyau MR, Adolph A, Vohra FA, Butte NF. Validation and calibration of physical activity monitors in children. Obes Res. 2002;10:150-157. PubMed doi:10.1038/oby.2002.24

34. Freedson PS, Melanson E, Sirard J. Calibration of the Computer Science and Applications, Inc. accelerometer. Med Sci Sports Exerc. 1998;30:777-781. PubMed doi:10.1097/00005768-199805000-00021

35. Straker L, Campbell A. Translation equations to compare ActiGraph GT3X and Actical accelerometers activity counts. BMC Med Res Methodol. 2012;12:54. PubMed doi:10.1186/1471-2288-12-54

36. Hagströmer M, Oja P, Sjostrom M. Physical activity and inactivity in an adult population assessed by accelerometry. Med Sci Sports Exerc. 2007;39:1502-1508. PubMed doi:10.1249/mss.0b013e3180a76de5

37. Pollock ML, Gaesser GA, Butcher JD, et al. American College of Sports Medicine Position Stand. The recommended quantity and quality of exercise for developing and maintaining cardiorespiratory and muscular fitness, and flexibility in healthy adults. Med Sci Sports Exerc. 1998;30:975-991.PubMeddoi:10.1097/00005768-19980600000032

38. Santana MJ, Feeny D, Ghosh S, Nador RG, Weinkauf J, Jackson K, Schafenacker M, Zuk D, Hubert G, Lien D. The construct validity of the health utilities index mark 3 in assessing health status in lung transplantation. Health Qual Life Outcomes. 28;8:110.

39. Toomingas A, Gavhed D. Workstation layout and work postures at call centres in Sweden in relation to national law, EU-directives and ISO-standards, and to operators' comfort and symptoms. Int J Ind Ergon. 2008;38:10511061. doi:10.1016/j.ergon.2008.02.010

40. Loomis D, Kromhout H. Exposure variability: concepts and applications in occupational epidemiology. Am J Ind Med. 2004;45:113-122. PubMed doi:10.1002/ajim. 10324

41. Mathiassen SE, Nordander C, Svendsen SW, Wellman HM, Dempsey PG. Task-based estimation of mechanical job exposure in occupational groups. Scand J Work 
Environ Health. 2005;31:138-151. PubMed doi:10.5271/ sjweh.861

42. Mathiassen SE, Burdorf A, van der Beek AJ. Statistical power and measurement allocation in ergonomic intervention studies assessing upper trapezius EMG amplitude. A case study of assembly work. $J$ Electromyogr Kinesiol. 2002;12:45-57. PubMed doi:10.1016/ S1050-6411(01)00028-1
43. Veiersted KB, Westgaard RH, Andersen P. Electromyographic evaluation of muscular work pattern as a predictor of trapezius myalgia. Scand J Work Environ Health. 1993;19:284-290. PubMed doi:10.5271/sjweh.1472

44. Mork PJ, Westgaard RH. Low-amplitude trapezius activity in work and leisure and the relation to shoulder and neck pain. J Appl Physiol. 2006;100:1142-1149. PubMed doi:10.1152/japplphysiol.01111.2005 